\title{
Valley Filtering in Spatial Maps of Coupling between Silicon Donors and Quantum Dots
}

\author{
J. Salfi, ${ }^{1}$ B. Voisin, ${ }^{1}$ A. Tankasala, ${ }^{2}$ J. Bocquel, ${ }^{1}$ M. Usman, ${ }^{3}$ M. Y. Simmons, ${ }^{1}$ \\ L. C. L. Hollenberg, ${ }^{3}$ R. Rahman, ${ }^{2}$ and S. Rogge ${ }^{1}$ \\ ${ }^{1}$ Centre for Quantum Computation and Communication Technology, School of Physics, \\ The University of New South Wales, Sydney, New South Wales 2052, Australia \\ ${ }^{2}$ Purdue University, West Lafayette, Indiana 47906, USA \\ ${ }^{3}$ Centre for Quantum Computation and Communication Technology, School of Physics, \\ University of Melbourne, Parkville, Victoria 3010, Australia
}

(Received 5 July 2017; revised manuscript received 14 June 2018; published 27 August 2018)

\begin{abstract}
Exchange coupling is a key ingredient for spin-based quantum technologies since it can be used to entangle spin qubits and create logical spin qubits. However, the influence of the electronic valley degree of freedom in silicon on exchange interactions is presently the subject of important open questions. Here we investigate the influence of valleys on exchange in a coupled donor-quantum-dot system, a basic building block of recently proposed schemes for robust quantum information processing. Using a scanning tunneling microscope tip to position the quantum dot with sub-nm precision, we find a near monotonic exchange characteristic where lattice-aperiodic modulations associated with valley degrees of freedom comprise less than $2 \%$ of exchange. From this we conclude that intravalley tunneling processes that preserve the donor's $\pm x$ and $\pm y$ valley index are filtered out of the interaction with the $\pm z$ valley quantum dot, and that the $\pm x$ and $\pm y$ intervalley processes where the electron valley index changes are weak. Complemented by tight-binding calculations of exchange versus donor depth, the demonstrated electrostatic tunability of donor-quantum-dot exchange can be used to compensate the remaining intravalley $\pm z$, oscillations to realize uniform interactions in an array of highly coherent donor spins.
\end{abstract}

DOI: 10.1103/PhysRevX.8.031049

Following proposals for spin-based quantum computing $[1,2]$, spin qubits have been demonstrated in, e.g., diamond [3], GaAs [4-7], Si donors [8], and Si quantum dots (QDs) [9-12]. Exchange coupling plays a key role in these proposals $[1,2]$ and has been employed experimentally to couple spins over short distances [5,11], and to define multispin qubits $[4,6,7,9,10,12]$ that can be coupled over larger distances via electric interactions [6], as also expected for spin-orbit qubits [13-17]. Because of the importance of exchange interactions, the impact of silicon's valley degree of freedom on electron tunneling and exchange has been the subject of many theoretical studies [18-28]. Notably, small changes in donor position [18-21,24-26] and QD surface roughness [27,28] are expected to produce large modulations of exchange coupling, affecting two-qubit gate fidelities, owing to the lattice aperiodicity of the valley wave vector. For donors, the negative effects of the predicted rapid nonmonotonic

Published by the American Physical Society under the terms of the Creative Commons Attribution 4.0 International license. Further distribution of this work must maintain attribution to the author(s) and the published article's title, journal citation, and DOI.
Subject Areas: Condensed Matter Physics,

Quantum Physics, Spintronics dependence of exchange could be reduced by atomic precision placement $[29,30]$ and/or quantum control schemes to recover two-qubit gate infidelities [22,23]. While predictions of the amplitude of the nonmonotonic oscillations vary significantly [18-28], experimentally establishing the strength of the exchange modulations has proven a difficult task and experimentally probing the role valleys in exchange has received no direct attention.

Tunneling and exchange in coupled donor-QD systems [31-34] underpin some recent theory proposals for robust spin-based quantum computing [35-37] seeking to exploit the long donor spin coherence times [38-40] without direct exchange between donors. The role of valleys in coupled donor-QD systems differs compared to the well-studied case of two donors [30,41,42]: the absence of $\pm x$ and $\pm y$ valleys in the two-valley $( \pm z)$ QD state means that intravalley exchange processes, where electrons preserve their valley index, occur for the $\pm z$ valleys but not for $\pm x$ and $\pm y$ valleys of the donor. Though not yet observed experimentally, this filtering of $\pm x$ and $\pm y$ valley degrees of freedom from intravalley donor-QD exchange should eliminate the main source rapid nonmonotonic variations of exchange with in-plane donor position. However, weaker intervalley processes where electrons change their 
valley index $[43,44]$ remain a potential source of rapid nonmonotonic exchange variations. Ignored in exchange calculations to date [18-25], intervalley processes become stronger as wave functions get smaller [43,44]. Large variations in exchange are also expected with donor depth variations, due to $\pm z$ intravalley tunneling, where the phase of the donor (QD) electron is pinned by the ion (interface). The extent to which these processes influence schemes for donor-QD-based quantum computing has yet to be established [35-37].

Here we experimentally investigate whether changes in lateral QD position can overcome variations of donor-QD exchange associated with high spatial frequency "valley" oscillations and slow envelope function decay, for threedimensional donor positioning uncertainty. This is accomplished by experimentally probing the exchange coupling $J$ of a donor bound electron with a highly localized electrostatic QD whose lateral position $\mathbf{R}$ relative to the donor can be controlled with sub-nm precision. The single-electron QD, which has a large $\sim 12 \mathrm{meV}$ charging energy and correspondingly few-nm small spatial extent, is formed beneath a passivated Si surface and its position, and coupling to donors, is controlled by moving the tip laterally above the sample surface. Here, we measure the donor-QD energy spectrum by single-electron transport $[45,46]$ to quantify the strength of the intervalley interference processes in the exchange coupling $J(\mathbf{R})$. We note that the tunability of the exchange interaction opens up interesting possibilities to electrically probe small-scale dopant-based quantum simulators [47-49], or to perform electrical spin readout on optically active impurity centers in materials like silicon carbide [50,51], silicon [52,53], and diamond [54,55].

We find that lattice-aperiodic exchange has a small amplitude $\lesssim 2 \%$ of the nominal exchange coupling, evidencing the valley filtering effect and setting an upper bound on the intervalley tunneling strength. We also experimentally explore the tunability of the donor-QD exchange with $\mathbf{R}$, and find that a modest 6-nm lateral QD shift changes the donorQD exchange by an order of magnitude. Finally, we show that the QD also has a negligible impact on the electronic orbital and valley population of the donor, which is $3.6 \mathrm{~nm}$ beneath the $\mathrm{Si}$ surface, which is important for some proposals [35,36]. Using $s p^{3} d^{5} s^{*}$ calculations, we find that the observed tunability of donor-QD exchange can readily compensate variations in exchange due to $\mathrm{nm}$-scale donor depth uncertainty. These results show that valley-induced variations in donor-QD exchange can be (i) altogether neglected for in-plane donor positioning variations due to the valley filtering effect and weak intervalley scattering and (ii) compensated for donor depth variations by modest electrostatic tuning of QD wave functions using surface gates [35-37], without distorting the donor wave function.

The key ingredient in our experiment is a single-electron QD whose coupling to individual donors can be tuned by controlling the QD position with sub-nm precision, using a scanning tunneling microscope (STM) tip [Fig. 1(a)]. The QD state is formed below a silicon-vacuum surface when the bands are locally bent downwards by the tip due to a bias $V$ applied to a reservoir [Fig. 1(b)] [56,57]. The QD and donor are contained in a lightly doped region, above a highly doped reservoir and below a (100) hydrogen-terminated surface [Fig. 1(a)]. The doping gradient was prepared by thermal annealing [45].

The energy of the QD was probed by spatially resolved single-electron tunneling. For the data shown in the $d I / d V$ map of Fig. 1(c) containing a neutral donor resonance $[45,59]$ at $V \approx-0.70 \mathrm{~V}$, we identify the first electron in a tip-induced QD state when the bands are bent downward for the resonance at $V \approx-1.10 \mathrm{~V}$, away from the donor. Notably, the resonance shifts to $V \approx-1.05 \mathrm{~V}$ as the QD approaches the neutral donor, showing that the coupled donor-QD state has a lower energy than the isolated donor and QD, since less downward bias of the localized state (relative to the reservoir) is required for resonant tunneling.

The observed local dip of the QD resonance near a neutral donor in Fig. 1(c) is inconsistent with a noninteracting state of the donor and QD where the QD energy would not depend on tip position [Fig. 1(d), lower black line]. To explain the data we need to consider spin-singlet paired two-electron states $S(i, j)$ with $i$ electrons on the donor and $j$ on the QD. Charging an isolated donor with a second electron can also be ruled out, since a parabolic $S(2,0)$ resonance would be expected in this case [Fig. 1(d), upper black line]. This is because as the tip moves away from the donor it is less effective at locally influencing the potential at the donor site [60] so a larger bias is needed to overcome the donor's on-site Coulomb repulsion. In contrast, the resonance in Fig. 1(c) flattens out, approaching $S(1,1)$-like behavior. Consequently, the two-electron (2e) state in Fig. 1(c) can only be understood as a hybridized superposition of $S(1,1)$ and $S(2,0)$ singlets [Fig. 1(d), solid blue line].

Importantly, the donor-QD system forms a molecular state in Fig. 1(c) since the donor-QD exchange energy $J$ well exceeds the reservoir tunnel rates, $h\left(\Gamma_{\text {in }}+\Gamma_{\text {out }}\right)$. This result is obtained by combining two experimentally established inequalities. First, $d I / d V$ has a line shape of a thermally broadened reservoir [Fig. 2(a)], so $k_{B} T>h\left(\Gamma_{\text {in }}+\Gamma_{\text {out }}\right)$. Second, $J$ well exceeds $k_{B} T$, as we show later.

Using the spatially resolved map of $1 e \rightarrow 0 e$ tunneling from the donor at $V=-0.80 \mathrm{~V}$ [Fig. 2(b)], we identify the donor ground state $[59,61]$ by the $A_{1}$ valley interference pattern [45]. We determine the donor depth $6.75 a_{0}$ beneath the silicon surface $\left(a_{0}=0.543 \mathrm{~nm}\right)$ using a comprehensive tunneling image analysis [62]. We assume zero electric field for comparison with Fig. 2(b), which is justified since the tip bias $V=-0.80 \mathrm{~V}$ induces a small electric field $-1 \pm 2 \mathrm{MV} / \mathrm{m}$ [63]. With the $2 e$ donor-QD state in the bias window at $V=-1.125 \mathrm{~V}$, the electric field in the sample is considerably larger $(13 \pm 1 \mathrm{MV} / \mathrm{m}$; see Ref. [63]). With the second resonance in the bias window, 
(a)
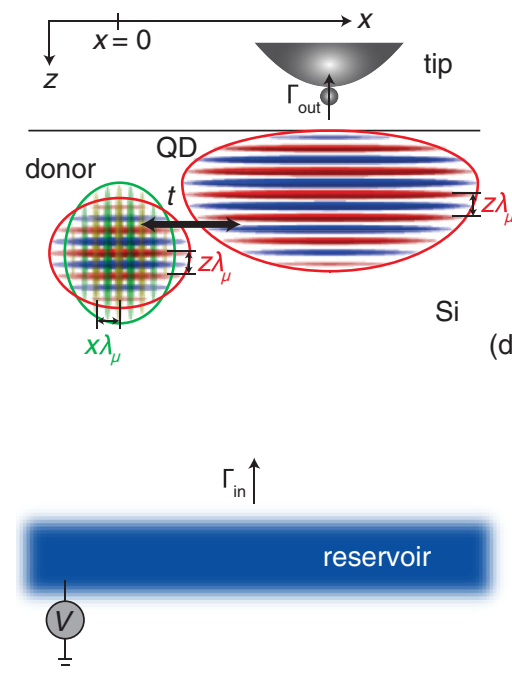

(b)

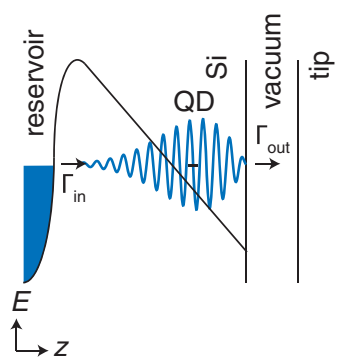

(d)
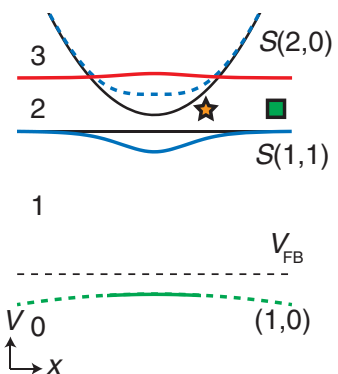

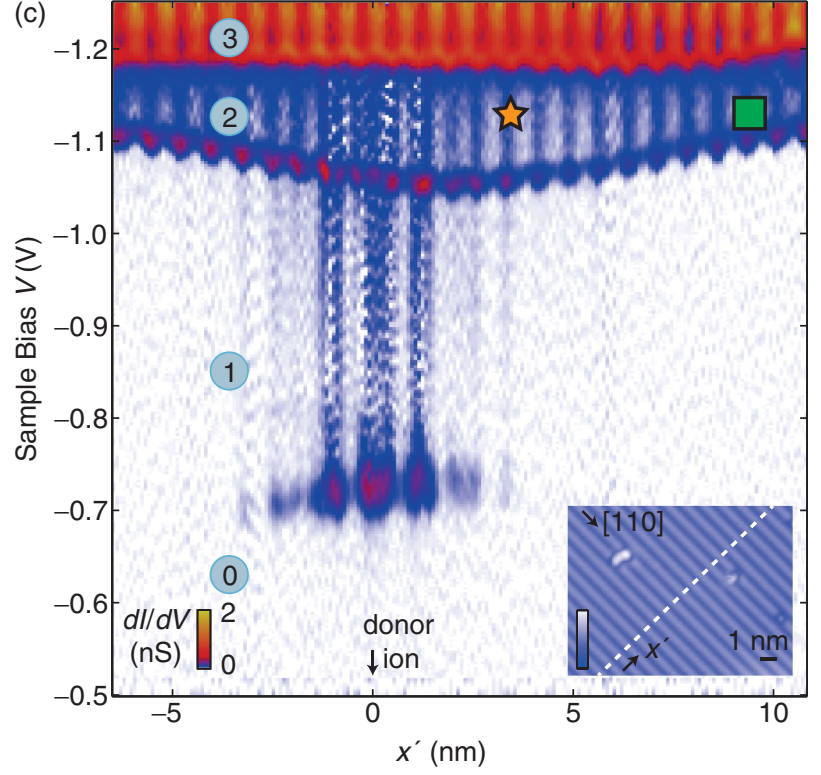

FIG. 1. Schematic of single-electron tunneling from buried reservoir, to coupled donor-QD state, to the tip. The donor wave function parameters are taken from Ref. [58], and the $\pm x$ and $\pm z$ valleys participating in the six-valley superposition are labeled. The $\pm y$ valleys of the donor are omitted for clarity. $\lambda_{\mu}=2 \pi / k_{\mu}$, where $k_{\mu}=0.82\left(2 \pi / a_{0}\right)$ is the conduction band minimum wave vector, and $a_{0}$ is silicon's lattice constant. (b) Schematic diagram of isolated QD resonance created by tip-induced band bending. (c) Measured $d I / d V$ spectrum labeling the $0,1,2$ (donor-QD hybrid) and 3 electron regions along the 110 crystal direction. Inset: Measured topography where dashed line shows locations for $d I / d V$ data. (d) Energy diagram for transitions between $0,1,2$, and 3 electron states labeled with green, blue, and red lines, respectively. Tunnel coupling $t$ between the donor and QD hybridizes the two-electron states $S(2,0)$ and $S(1,1)$, where $S(i, j)$ is the singlet with $i$ electrons on the donor and $j$ on the QD. Unhybridized two-electron states are shown as black lines and hybridized states as blue lines. The star (box) denotes the QD position for strong (weak) hybridization of two-electron states. Temperature is $4.2 \mathrm{~K}$.

spatially resolved electron tunneling to the tip [Fig. 2(c)] represents a $2 e \rightarrow 1 e$ quasiparticle wave function (QPWF) [47,64-66].

For the discussion of the measured two-electron donor-QD hybrid QPWF resonance in Fig. 2(c), we use the spin singlet model illustrated in Fig. 1(d) with $|S\rangle=c_{1,1}|S(1,1)\rangle+c_{2,0}|S(2,0)\rangle$, where $c_{i, j}$ is the probability amplitude for $|S(i, j)\rangle$. For the $2 e \rightarrow 1 e$ transition, the tunneling current is $I(\mathbf{r})=\left|\mathbb{D}\left[2^{-1 / 2} c_{1,1} \psi_{d_{1}}(\mathbf{r})\right]\right|^{2}+$ $\left|\mathbb{D}\left[2^{-1 / 2} c_{1,1} u_{q}(\mathbf{r})+c_{2,0} \psi_{d_{2}}(\mathbf{r})\right]\right|^{2}[63]$. Here, $\psi_{d_{i}}(\mathbf{r})$ is the donor orbital for electron number $i, \mathbf{r}$ is the movable QD's position relative to the donor, $u_{q}(\mathbf{r})$ is the lattice-scale structure of the moving QD's wave function, and $\mathbb{D}$ is a differential operator that takes the STM tip orbital into account [67]. We have found exceptionally good agreement of our single donor measurements $[45,46]$ with $s p^{3} d^{5} s^{*}$ theory, including $d$-orbital tips [62].

As expected, the center of the donor-QD QPWF map [Fig. 2(c)] strongly resembles the measured neutral donor [Fig. 2(b)] because both $S(2,0)$ and $S(1,1)$ contain donor bound orbitals, as reflected in the above expression for $I(\mathbf{r})$. We note that when the $2 e \rightarrow 1 e$ transition is in the bias window, the $1 e \rightarrow 0 e$ transition also remains energetically allowed [Fig. 2(c)]. However, following the $2 e \rightarrow 1 e$ transition, the $1 e \rightarrow 0 e$ transition is much less likely than a $1 e \rightarrow 2 e$ transition because the electron loading rate from the reservoir $\Gamma_{\text {in }}=\Gamma_{1 e \rightarrow 2 e}$ far exceeds the tunnel rate to the tip $\Gamma_{\text {out }}=\Gamma_{1 e \rightarrow 0 e}$ [63]. Hence, the strong appearance of the donor in the QD resonance of Fig. 2(c) is not due to a $1 e \rightarrow 0 e$ transition. Rather, it confirms the pairing interaction of the QD with the donor.

Away from the donor, the donor-QD resonance [Fig. 2(c)] is lattice periodic in the $(x, y)$ plane as expected for a QD wave function containing only $+z$ and $-z$ valleys $[68,69]$. Importantly, the QD and donor states are expected to have a significant vertical overlap as illustrated in Fig 1(a), since the QD charge density is expected to peak at $z_{0} \approx 3.5 a_{0}$, just $\sim 2 \mathrm{~nm}$ from the donor ion at $6.75 a_{0}$. Here, $z_{0}$ was estimated using the triangular well approximation [68] with $z_{0} \approx 1.32\left(\hbar^{2} / 2 m e \mathcal{E}_{z}\right)^{1 / 3}$, where $\mathcal{E}_{z}=13 \pm 2 \mathrm{MV} / \mathrm{m}$ is the electric field and $m$ is the longitudinal electron effective mass in $\mathrm{Si}$ [63].

The remainder of the analysis focuses on the dependence of the envelope and spatial oscillations present in the donorQD resonance energy $E\left(x^{\prime}\right)$. Plotted in Fig. 3(a), $E\left(x^{\prime}\right)$ obtained from Fig. 1(c) and the extracted lever arm [63] varies by $5.5 \mathrm{meV}$ over a $16-\mathrm{nm}$ range of tip positions. Notably, the interface experienced by the QD is atomically 


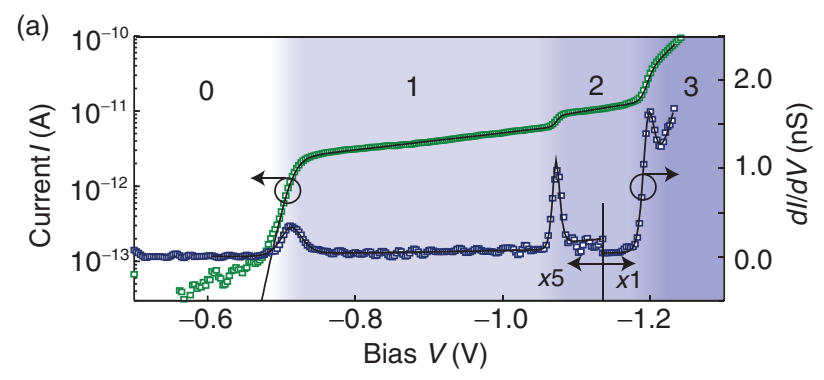

(b) $1 e / 0 e, V=-0.800 \mathrm{~V}$

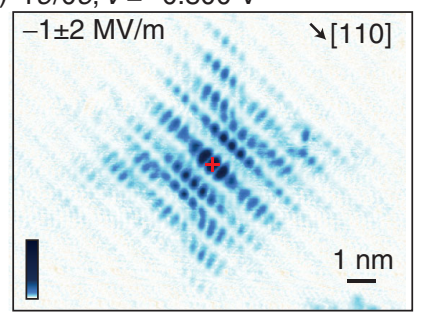

(c) $2 e / 1 e, V=-1.125 \mathrm{~V}$
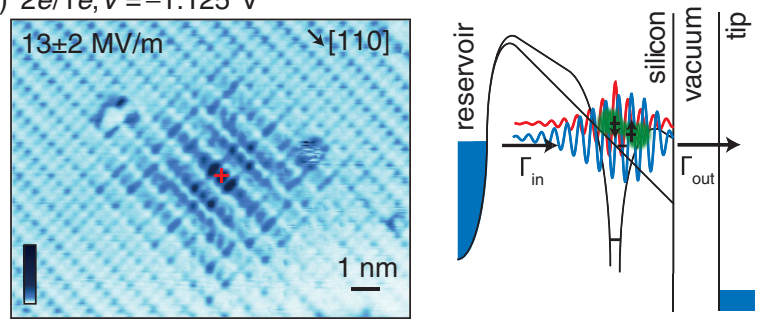

FIG. 2. (a) Bias dependence of current $I$ (green) and conductance $d I / d V$ (blue) over the donor, taken at $-1.42 \mathrm{~nm}$ in Fig. 1(c), and fit to rate-equation model (black lines). (b) Measured current map of the neutral donor. Right: Tunnel junction energy diagram. (c) Measured current map of coupled donor-QD singlet. Right: Tunnel junction energy diagram. Data for the donor and donorQD resonances were acquired in the spatial region of the inset of Fig. 1(c). The donor ion position is marked with a red cross.

flat [Fig. 1(c), inset], which is important since roughness disturbs the valley phase and exchange coupling of QDs [27,28,70-74]. The donor-QD energy map $E\left(x^{\prime}\right)$ is dominated by exchange coupling $J\left(x^{\prime}\right)=J_{d q}\left(x^{\prime}\right)+$ $\frac{1}{2}\left(U_{e 1}-\sqrt{8 t\left(x^{\prime}\right)^{2}+U_{e 1}^{2}}\right)$, which in turn contains tunneling and exchange terms $t\left(x^{\prime}\right)$ and $J_{d q}\left(x^{\prime}\right)$ [19]. Here, $U_{e 1}=$ $E_{d}-E_{q}+U_{d d}-U_{d q}$ is an effective addition energy, where $U_{d d}$ is the donor charging energy and $U_{d q}$ is the QD-donor electron repulsion. The remaining contribution to $E\left(x^{\prime}\right)$ is the Coulomb interaction of the QD with the neutral donor, estimated to be $\sim 1 \mathrm{meV}$ [63]. Importantly, the Fourier decomposition of $E\left(x^{\prime}\right)$ [Fig. 3(b)] contains no lattice-aperiodic components above $\approx 1 \%$ of the average of $E\left(x^{\prime}\right)\left(\right.$ at $\left.q_{110}=0\right)$. Given that $J\left(x^{\prime}\right)$ comprises more than $50 \%$ of $E\left(x^{\prime}\right)$ and the residual Coulomb interactions in $E\left(x^{\prime}\right)$ do not have lattice aperiodic components, the $1 \%$ upper bound for $E\left(x^{\prime}\right)$ corresponds to a $2 \%$ upper bound of lattice-aperiodic components of $J\left(x^{\prime}\right)$.

Tunneling and exchange in a coupled donor-QD system differs from two donors $[18,19,21,24-26]$ because the QD
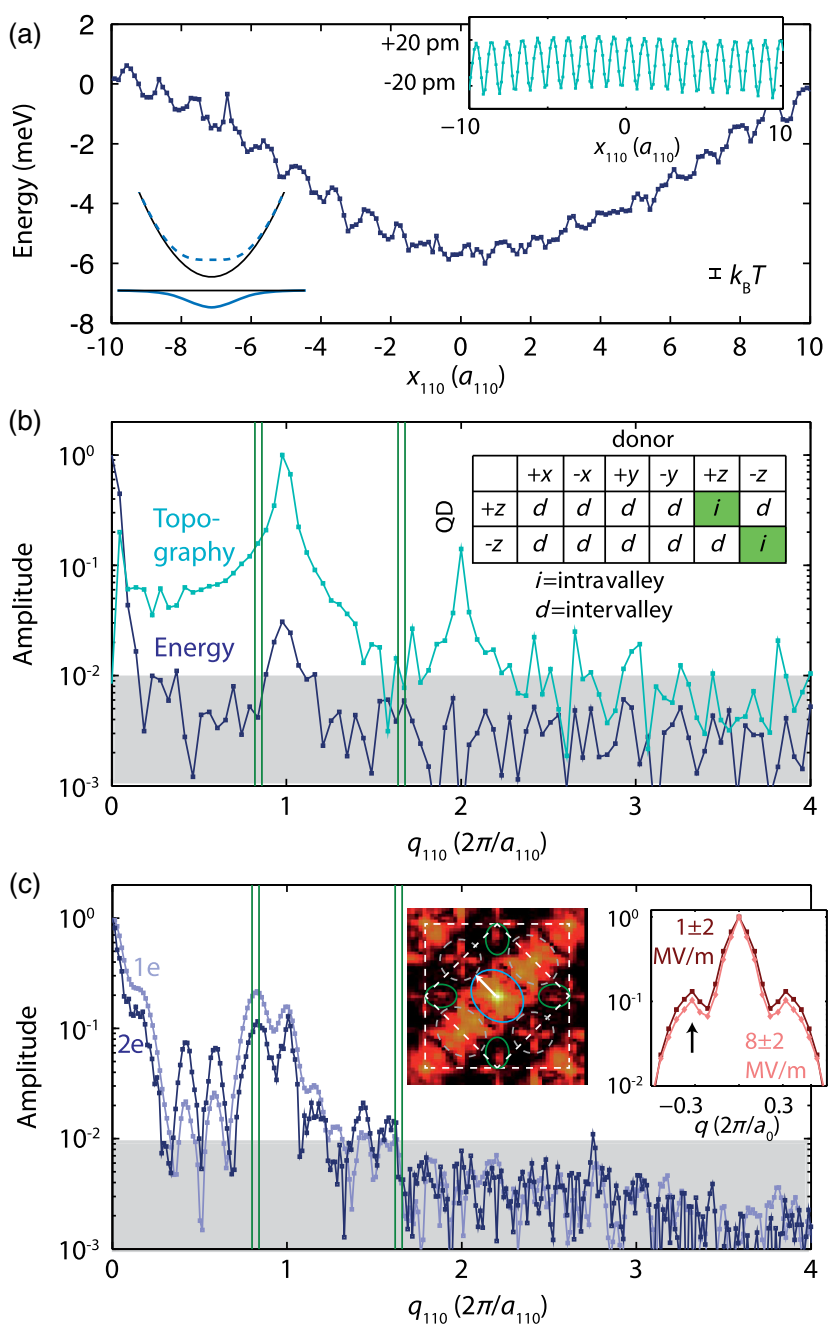

FIG. 3. (a) Donor-QD energy $E\left(x^{\prime}\right)$ as a function of tip position $x$. Inset: Measured topography. (b) Spatial Fourier decomposition of $E\left(x^{\prime}\right)$ where lattice-aperiodic components are less than $1 \%$ of value at $q_{110}=0$. Inset: Intravalley (green) and intervalley (white) tunneling for donor and QD. (c) One-dimensional spatial Fourier decomposition of $2 e$ and $1 e$ tunneling current along a [110] direction, with lattice-aperiodic oscillations due to valley superpositions in donor bound states at $q_{110}=0.85\left(2 \pi / a_{110}\right)$ and harmonics, where $a_{110}=\sqrt{2} a_{0}$. Left-hand inset: Twodimensional Fourier decomposition of one-electron tunneling probability at electric field $8 \pm 2 \mathrm{MV} / \mathrm{m}$ and bias $V=$ -1.010 V. Right-hand inset: Line cut through two-dimensional decomposition evidencing negligible valley repopulation of donor just below the $2 e$ resonance.

is a superposition of $\pm z$ valleys only, and the donor is a six-valley superposition as evidenced by lattice-aperiodic components in the Fourier transforms of STM tunnel current maps [Fig. 3(c)]. Notably, the six-valley superposition of the $6.75 a_{0}$ deep donor is hardly affected at all by the QD electrostatic potential, even at an applied electric field of $8 \pm 2 \mathrm{MV} / \mathrm{m}$ below the $2 e$ resonance $(V=-1.010 \mathrm{~V})$. This is evidenced by the Fourier decomposition of the donor measurements below the $2 e$ resonance 
[Fig. 3(c), left-hand inset]. The amplitude of the Fourier peak at $\mathbf{q}= \pm(+0.15,-0.15)\left(2 \pi / a_{0}\right)$ [black arrow in the right-hand inset of Fig. 3(c)] reflects the $x$ and $y$ valley population [45,58], and depends very little on the tip bias $V$ in our experiment [Fig. 3(c)]. By extending our theory comparison [62] to include electric fields, we estimate a $0.5 \%$ change in the population of the $x$ and $y$ valleys with the increase in electric field from $-1 \pm 2 \mathrm{MV} / \mathrm{m}$ to $8 \pm$ $2 \mathrm{MV} / \mathrm{m}$ due to the STM tip voltage [63]. This is important because for shallower donors where ion-implant statistical uncertainty are suppressed [75], hybrid donor-QD systems can be formed with negligible perturbation to donor valley composition and hyperfine coupling.

We note that $E\left(x^{\prime}\right)$ contains a lattice-periodic oscillation [Figs. 3(a) and 3(b)]. This oscillation is likely an artifact from QD energy and wave function changes induced by tipheight variation $\delta z$ [Fig. 3(a), inset]. For the former, an energy shift of $\delta E=e \alpha \delta z\left(\epsilon_{\mathrm{Si}} \mathcal{E}_{z}\right)$ is expected [46], where $\alpha \approx 0.1$ is the lever arm from our fit. For $\delta z=40 \mathrm{pm}$ [Fig. 3(a), inset], $\delta E=0.6 \mathrm{meV}$, in agreement with the measured $0.7 \mathrm{meV}$ oscillation. Notably, the smooth exchange variation in Fig. 3(a) indicates that the valley phase $[28,72,73]$ varies little, even though the electric field varies by $\sim 3 \mathrm{MV} / \mathrm{m}$ due to the change in the resonance voltage from $V=-1.05 \mathrm{~V}$ to $V=-1.10 \mathrm{~V}$.

We now consider lattice-aperiodic oscillations due to interference of valley degrees of freedom in coupled donorQD systems. The spectral decomposition of $J(\mathbf{R})$ can be theoretically understood from an extended Hubbard model for donor-QD tunneling, $t(\mathbf{R})=\left\langle\psi_{q}\left|v_{q}+v_{d}\right| \psi_{d}\right\rangle$, and exchange, $J_{d q}(\mathbf{R})=\left\langle\psi_{d} \psi_{q}\left|e^{2}\left(4 \pi \epsilon\left|\mathbf{r}_{1}-\mathbf{r}_{2}\right|\right)^{-1}\right| \psi_{q} \psi_{d}\right\rangle$. Here, $\mathbf{R}$ is the donor-QD separation, $v_{d(q)}$ is the donor (QD) potential, $\psi_{d}(\mathbf{r})=\sum_{\mu} \psi_{d \mu}(\mathbf{r})\left[\psi_{q}(\mathbf{r})=\sum_{\mu= \pm z} \psi_{q \mu}(\mathbf{r})\right]$ is the six-valley donor (two-valley QD) wave function and $\mu$ is the valley index. In $J(\mathbf{R})$, intravalley (valley preserving) and intervalley (valley modifying) terms have latticeaperiodic prefactors $\exp (i \mathbf{k} \cdot \mathbf{R})$, since $\mathbf{k}$ values are distributed about the conduction band minima. While the intravalley tunneling process present in interdonor exchange can be evaluated readily [19], it is expected to be absent here for $x$ and $y$ valleys since they are not present in the QD state. The remaining intervalley processes where electrons change valley index while tunneling [43,44] contribute lattice-aperiodic terms $\exp (i \mathbf{k} \cdot \mathbf{R})$ to $J(\mathbf{R})$. Hence, the $2 \%$ bound on the lattice-aperiodic exchange both reflects the effectiveness of valley filtering and provides an upper bound on the strength of intervalley exchange compared to total exchange, which, to our knowledge, has not been reported to date. In particular, the intervalley exchange falls outside the scope of the effective mass approximation, but is expected to be enhanced for localized states compared to extended states. This is relevant because the nm-spatial extent of the localized wave functions is similar to silicon's lattice constant [76].
The experimentally confirmed weakness of the intervalley tunneling means that lateral and vertical donor positioning uncertainty of donors will influence donor-QD exchange in different ways. Lateral donor positioning uncertainty will influence coupling predominantly through the nm-scale envelope decay length of the QD. In contrast, vertical donor positioning uncertainty will influence coupling through a combination of the vertical decay length of the donor and QD and interference processes in the intravalley exchange. The interference should contain an oscillatory term in donor depth because the surface pins the valleys of the QD while the ion pins the valleys of donor bound electron. The strategy that stands out to compensate these exchange variations is to adjust the QD confinement potential and therefore overlap of the QD state with the fixed donor. This is already accomplished in our experiment since the QD follows the potential of the STM tip, and in proposed devices could be realized by tuning surface gate voltages [36,37]. In particular, our measurements [Fig. 3(a)] show that a change of donor-QD separation by $6 \mathrm{~nm}$ changes donor-QD exchange by an order of magnitude, showing that strongly confined QDs allow for a tremendous exchange tuning range.

To determine if this tuning range is sufficient to overcome intravalley oscillations in exchange due to depth variations in the donors, a quantitative theory analysis has been carried out with atomistic $s p^{3} d^{5} s^{*}$ tight binding. Experimentally measuring these oscillations is difficult since it would require the ability to change the valley phase of the QD wave function, or directly measuring $<0.5 \mathrm{meV}$ values of exchange with direct transport, which is not possible in our scheme at $4.2 \mathrm{~K}$. The QD state in the calculation was calibrated so that full configuration interaction wave functions [26,77] reproduce experimentally measured spectra. A 5-nm STM tip radius was found to reproduce the bias where $0 e \rightarrow 1 e$ and $1 e \rightarrow 2 e \mathrm{QD}$ transitions occur, away from the donor. The lowering of the addition energy due to donor-QD coupling when the QD is directly over the donor is calculated to be $E\left(x^{\prime}=0\right)=6.8 \mathrm{meV}$, compared to the value $6 \mathrm{meV}$ in experiments [Fig. 3(a)].

The expected variation in exchange with vertical donor positioning uncertainty was estimated by computing the donor-QD exchange for a range of donor depths and QD distances. The geometry used in the calculation includes the tip potential and donor ion potential. A cross section of the calculated charge density including the donor $(x=0)$ and QD ( $x=8 \mathrm{~nm})$ is shown in Fig. 4(a). In agreement with our measurements, the exchange varies slowly with lateral QD position [Fig. 4(a), inset], showing that tight binding accurately reproduces the weak intervalley scattering observed in experiments. The calculated exchange varies rapidly with donor depth $z_{0}$ [Fig. 4(b)], but notably, the total variation including the rapidly varying intravalley interference and envelope decay is less than 2 orders of magnitude for depths between 2.2 and $3.7 \mathrm{~nm}$. This is 

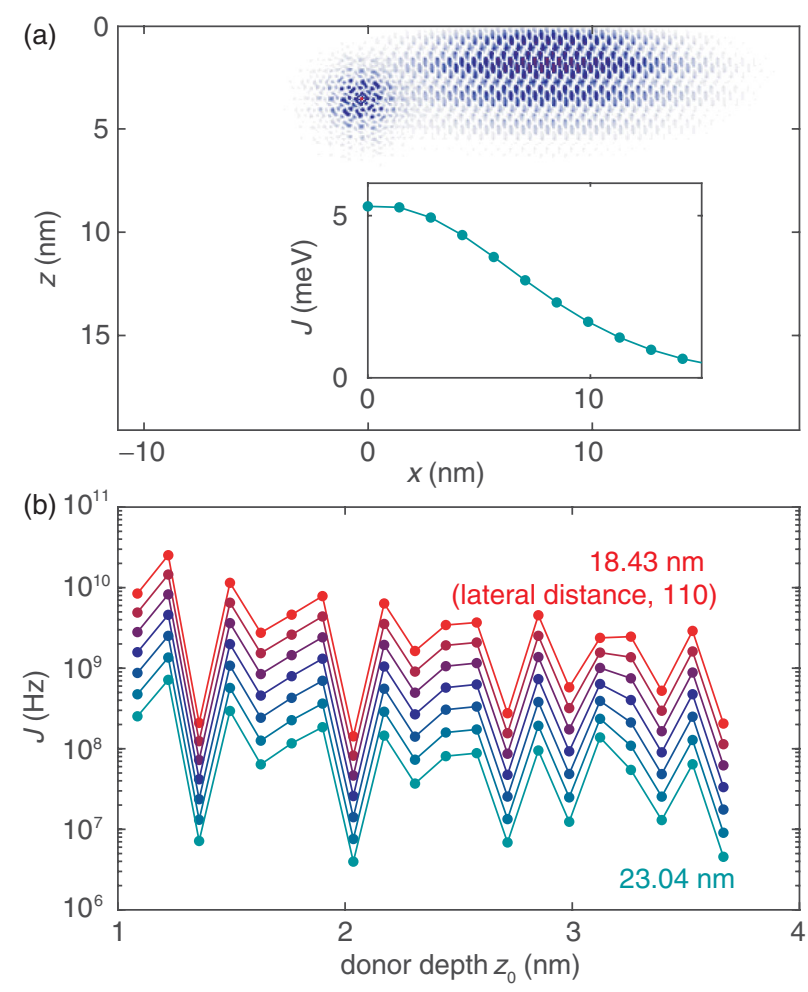

FIG. 4. (a) Calculated electron density for donor and quantum dot, where only the donor contains lattice incommensurate components. Inset: Predicted decay of exchange as a function of donor-QD distance. (b) Calculated dependence of donor-QD exchange on donor depth, for different displacements along 110 between 18.43 and $23.04 \mathrm{~nm}$.

important because it indicates that variations in exchange due to donor depth uncertainty can be compensated by adjusting donor-QD wave function overlap using gates. The calculations for different donor-QD lateral displacements along the 110 direction [Fig. 4(b)] show that a change of QD position of $\sim 4.5 \mathrm{~nm}$, between 18.43 and $23.04 \mathrm{~nm}$, is sufficient to overcome this variation. We also note that similar to interdonor exchange, residual coupling uncertainty can in principle be corrected by quantum control $[22,23]$.

In conclusion, we spatially mapped the energy of a neutral donor coupled to a single-electron QD that can be positioned in the plane with sub-nm accuracy using a STM tip. Besides additional applications of coupling to optically active impurities [50-55] or small-scale quantum simulators [47-49], our results highlight that, similar to predictions for donor-donor interactions in strained $\mathrm{Si}$, donor-QD interactions [34-37] do not suffer from valley-induced variations in exchange due to in-plane donor positioning uncertainty. The demonstrated monotonic tunability of donor-QD exchange with QD position is therefore promising for the realization of uniform exchange couplings between highly coherent donors using tunable, electrostatically defined QDs [35-37] that are compatible with an all-donor-based approach $[1,78]$.
The authors would like to thank D. Culcer for helpful discussions. We acknowledge support from the ARC Centre of Excellence for Quantum Computation and Communication Technology (CE110001027), and partial support from the U.S. Army Research Office (W911NF-081-0527). J. S. acknowledges support from an ARC DECRA fellowship (DE160101490). The authors acknowledge the use of computational resources from NanoHUB, the Network for Computational Nanotechnology at Purdue University, and from the Australian Government through the Pawsey Supercomputing Centre under the National Computational Merit Allocation Scheme (NCMAS). This work used the Extreme Science and Engineering Discovery Environment (XSEDE) ECS150001, which is supported by National Science Foundation Grant No. ACI-1548562 [79].

[1] B. E. Kane, A Silicon-Based Nuclear Spin Quantum Computer, Nature (London) 393, 133 (1998).

[2] D. Loss and D. P. DiVincenzo, Quantum Computation with Quantum Dots, Phys. Rev. A 57, 120 (1998).

[3] L. Childress, M. V. Gurudev Dutt, T. J. MM, A. S. Zibrov, F. Jelezko, J. Wrachtrup, P. R. Hemmer, and M. D. Lukin, Coherent Dynamics of Coupled Electron and Nuclear Spin Qubits in Diamond, Science 314, 281 (2006).

[4] J. R. Petta, A. C. Johnson, J. M. Taylor, E. A. Laird, A. Yacoby, M. D. Lukin, C. M. Marcus, M. P. Hanson, and A. C. Gossard, Coherent Manipulation of Coupled Electron Spins in Semiconductor Quantum Dots, Science 309, 2180 (2005).

[5] K. C. Nowack, M. Shafiei, M. Laforest, G. E. D. K. Prawiroatmodjo, L. R. Schreiber, C. Reichl, W. Wegscheider, and L. M. K. Vandersypen, Single-Shot Correlations and Two-Qubit Gate of Solid-State Spins, Science 333, 1269 (2011).

[6] M. D. Shulman, O. E. Dial, S. P. Harvey, H. Bluhm, V. Umansky, and A. Yacoby, Demonstration of Entanglement of Electrostatically Coupled Singlet-Triplet Qubits, Science 336, 202 (2012).

[7] J. Medford, J. Beil, J. M. Taylor, S. D. Bartlett, A. C. Doherty, E. I. Rashba, D. P. DiVincenzo, H. Lu, A. C. Gossard, and C. M. Marcus, Self-Consistent Measurement and State Tomography of an Exchange-Only Spin Qubit, Nat. Nanotechnol. 8, 654 (2013).

[8] J. J. Pla, K. Y. Tan, J. P. Dehollain, W. H. Lim, J. J. L. Morton, D. N. Jamieson, A. S. Dzurak, and A. Morello, A Single-Atom Electron Spin Qubit in Silicon, Nature (London) 489, 541 (2012).

[9] B. M. Maune, M. G. Borselli, B. Huang, T. D. Ladd, P. W. Deelman, K. S. Holabird, A. A. Kiselev, I. AlvaradoRodriguez, R. S. Ross, A. E. Schmitz, M. Sokolich, C. A. Watson, M. F. Gyure, and A. T. Hunter, Coherent SingletTriplet Oscillations in a Silicon-Based Double Quantum Dot, Nature (London) 481, 344 (2012).

[10] D. Kim, Z. Shi, C. B. Simmons, D. R. Ward, J. R. Prance, T. S. Koh, J. K. Gamble, D. E. Savage, M. G. Lagally, M. Friesen, S. N. Coppersmith, and M. A. Eriksson, Quantum Control and Process Tomography of a Semiconductor 
Quantum Dot Hybrid Qubit, Nature (London) 511, 70 (2014).

[11] M. Veldhorst, C. H. Yang, J. C. C. Hwang, W. Huang, J. P. Dehollain, J. T. Muhonen, S. Simmons, A. Laucht, F. E. Hudson, K. M. Itoh, A. Morello, and A. S. Dzurak, A TwoQubit Logic Gate in Silicon, Nature (London) 526, 410 (2015).

[12] M. D. Reed, B. M. Maune, R. W. Andrews, M. G. Borselli, K. Eng, M. P. Jura, A. A. Kiselev, T. D. Ladd, S. T. Merkel, I. Milosavljevic, E. J. Pritchett, M. T. Rakher, R. S. Ross, A. E. Schmitz, A. Smith, J. A. Wright, M. F. Gyure, and A. T. Hunter, Reduced Sensitivity to Charge Noise in Semiconductor Spin Qubits via Symmetric Operation, Phys. Rev. Lett. 116, 110402 (2016).

[13] C. Flindt, A. S. Sørensen, and K. Flensberg, Spin-Orbit Mediated Control of Spin Qubits, Phys. Rev. Lett. 97, 240501 (2006).

[14] K. C. Nowack, F. H. L. Koppens, Yu V. Nazarov, and L. M. K. Vandersypen, Coherent Control of a Single Electron Spin with Electric Fields, Science 318, 1430 (2007).

[15] S. Nadj-Perge, S. M. Frolov, E. P. A. M. Bakkers, and L. P. Kouwenhoven, Spin-Orbit Qubit in a Semiconductor Nanowire, Nature (London) 468, 1084 (2010).

[16] J. Salfi, J. A. Mol, Dimitrie Culcer, and S. Rogge, ChargeInsensitive Single-Atom Spin-Orbit Qubit in Silicon, Phys. Rev. Lett. 116, 246801 (2016).

[17] J. Salfi, M. Tong, S. Rogge, and D. Culcer, Quantum Computing with Acceptor Spins in Silicon, Nanotechnology 27, 244001 (2016).

[18] P. R. Cullis and J. R. Marko, Determination of the Donor Pair Exchange Energy in Phosphorus-Doped Silicon, Phys. Rev. B 1, 632 (1970).

[19] B. Koiller, X. Hu, and S. Das Sarma, Exchange in SiliconBased Quantum Computer Architecture, Phys. Rev. Lett. 88, 027903 (2001).

[20] B. Koiller, X. Hu, and S. D. Sarma, Strain Effects on Silicon Donor Exchange: Quantum Computer Architecture Considerations, Phys. Rev. B 66, 115201 (2002).

[21] C. Wellard and L. Hollenberg, Donor Electron Wave Functions for Phosphorus in Silicon: Beyond EffectiveMass Theory, Phys. Rev. B 72, 085202 (2005).

[22] M. J. Testolin, C. D. Hill, C. J. Wellard, and L. C. L. Hollenberg, Robust Controlled-NOT Gate in the Presence of Large Fabrication-Induced Variations of the Exchange Interaction Strength, Phys. Rev. A 76, 012302 (2007).

[23] C. D. Hill, Robust Controlled-not Gates from Almost Any Interaction, Phys. Rev. Lett. 98, 180501 (2007).

[24] G. Pica, B. W. Lovett, R. N. Bhatt, and S. A. Lyon, Exchange Coupling between Silicon Donors: The Crucial Role of the Central Cell and Mass Anisotropy, Phys. Rev. B 89, 235306 (2014).

[25] J. K. Gamble, N. T. Jacobson, E. Nielsen, A. D. Baczewski, J. E. Moussa, I. Montaño, and R. P. Muller, Multivalley Effective Mass Theory Simulation of Donors in Silicon, Phys. Rev. B 91, 235318 (2015).

[26] Y. Wang, A. Tankasala, L. C. L. Hollenberg, G. Klimeck, M. Y. Simmons, and R. Rahman, Highly Tunable Exchange in Donor Qubits in Silicon, npj Quantum Inf. 2, 16008 (2016).
[27] E. Nielsen, R. Rahman, and R. P. Muller, A Many-Electron Tight Binding Method for the Analysis of Quantum Dot Systems, J. Appl. Phys. 112, 114304 (2012).

[28] N. M. Zimmerman, P. Huang, and D. Culcer, Valley Phase and Voltage Control of Coherent Manipulation in Si Quantum Dots, Nano Lett. 17, 4461 (2017).

[29] M. Fuechsle, J. A. Miwa, S. Mahapatra, H. Ryu, S. Lee, O. Warschkow, L. C. L. Hollenberg, G. Klimeck, and M. Y. Simmons, A Single-Atom Transistor, Nat. Nanotechnol. 7, 242 (2012).

[30] B. Weber, Y. H. M. Tan, S. Mahapatra, T. F. Watson, H. Ryu, R. Rahman, L. C. L. Hollenberg, G. Klimeck, and M. Y. Simmons, Spin Blockade and Exchange in CoulombConfined Silicon Double Quantum Dots, Nat. Nanotechnol. 9, 430 (2014).

[31] G. P. Lansbergen, R. Rahman, C. J. Wellard, I. Woo, J. Caro, N. Collaert, S. Biesemans, G. Klimeck, L. C. L. Hollenberg, and S. Rogge, Gate-Induced Quantum-Confinement Transition of a Single Dopant Atom in a Silicon FinFET, Nat. Phys. 4, 656 (2008).

[32] R. H. Foote, D. R. Ward, J. R. Prance, J. K. Gamble, E. Nielsen, B. Thorgrimsson, D. E. Savage, A. L. Saraiva, M. Friesen, S. N. Coppersmith, and M. A. Eriksson, Transport through an Impurity Tunnel Coupled to a Si/SiGe Quantum Dot, Appl. Phys. Lett. 107, 103112 (2015).

[33] M. Urdampilleta, A. Chatterjee, C. C. Lo, T. Kobayashi, J. Mansir, S. Barraud, A. C. Betz, S. Rogge, M. F. GonzalezZalba, and J. J.L. Morton, Charge Dynamics and Spin Blockade in a Hybrid Double Quantum Dot in Silicon, Phys. Rev. X 5, 031024 (2015).

[34] P. Harvey-Collard, N. T. Jacobson, M. Rudolph, J. Dominguez, G. A. Ten Eyck, J. R. Wendt, T. Pluym, J. K. Gamble, M. P. Lilly, M. Pioro-Ladrière, and M. S. Carroll, Coherent Coupling between a Quantum Dot and a Donor in Silicon, Nat. Commun. 8, 1029 (2017).

[35] V. Srinivasa, H. Xu, and J. M. Taylor, Tunable Spin-Qubit Coupling Mediated by a Multielectron Quantum Dot, Phys. Rev. Lett. 114, 226803 (2015).

[36] G. Pica, B. W. Lovett, R. N. Bhatt, T. Schenkel, and S. A. Lyon, Surface Code Architecture for Donors and Dots in Silicon with Imprecise and Nonuniform Qubit Couplings, Phys. Rev. B 93, 035306 (2016).

[37] G. Tosi, F. A. Mohiyaddin, V. Schmitt, S. Tenberg, R. Rahman, G. Klimeck, and A. Morello, Silicon Quantum Processor with Robust Long-Distance Qubit Couplings, Nat. Commun. 8, 450 (2017).

[38] A. M. Tyryshkin, S. Tojo, J. J. L. Morton, H. Riemann, N. V. Abrosimov, P. Becker, H.-J. Pohl, T. Schenkel, M. L. W. Thewalt, K. M. Itoh, and S. A. Lyon, Electron Spin Coherence Exceeding Seconds in High-Purity Silicon, Nat. Mater. 11, 143 (2012).

[39] M. Steger, K. Saeedi, M. L. W. Thewalt, J. J. L. Morton, H. Riemann, N. V. Abrosimov, P. Becker, and H. J. Pohl, Quantum Information Storage for over 180 s Using Donor Spins in a ${ }^{28} \mathrm{Si}$ "Semiconductor Vacuum," Science 336, 1280 (2012).

[40] G. Wolfowicz, A. M. Tyryshkin, R. E. George, H. Riemann, N. V. Abrosimov, P. Becker, H.-J. Pohl, M. L. W. Thewalt, S. A. Lyon, and J. J. L. Morton, Atomic Clock Transitions in Silicon-Based Spin Qubits, Nat. Nanotechnol. 8, 561 (2013). 
[41] J. P. Dehollain, J. T. Muhonen, K. Y. Tan, A. Saraiva, D. N. Jamieson, A.S. Dzurak, and A. Morello, Single-Shot Readout and Relaxation of Singlet and Triplet States in Exchange-Coupled ${ }^{31} \mathrm{P}$ Electron Spins in Silicon, Phys. Rev. Lett. 112, 236801 (2014).

[42] M. F. Gonzalez-Zalba, A. Saraiva, M. J. Calderón, D. Heiss, B. Koiller, and A.J. Ferguson, An Exchange-Coupled Donor Molecule in Silicon, Nano Lett. 14, 5672 (2014).

[43] T. H. Ning and C. T. Sah, Multivalley Effective-Mass Approximation for Donor States in Silicon. I. Shallow-Level Group-V Impurities, Phys. Rev. B 4, 3468 (1971).

[44] S. T. Pantelides and C. T. Sah, Theory of Localized States in Semiconductors. I. New Results Using an Old Method, Phys. Rev. B 10, 621 (1974).

[45] J. Salfi, J. A. Mol, R. Rahman, G. Klimeck, M. Y. Simmons, L. C. L. Hollenberg, and S. Rogge, Spatially Resolving Valley Quantum Interference of a Donor in Silicon, Nat. Mater. 13, 605 (2014).

[46] B. Voisin, J. Salfi, J. Bocquel, R. Rahman, and S. Rogge, Spatially Resolved Resonant Tunneling on Single Atoms in Silicon, J. Phys. Condens. Matter 27, 154203 (2015).

[47] J. Salfi, J. A. Mol, R. Rahman, G. Klimeck, M. Y. Simmons, L. C. L. Hollenberg, and S. Rogge, Quantum Simulation of the Hubbard Model with Dopant Atoms in Silicon, Nat. Commun. 7, 11342 (2016).

[48] N. H. Le, A. J. Fisher, and E. Ginossar, Extended Hubbard Model for Mesoscopic Transport in Donor Arrays in Silicon, Phys. Rev. B 96, 245406 (2017).

[49] A. Dusko, A. Delgado, A. Saraiva, and B. Koiller, Adequacy of Si:P Chains as Fermi-Hubbard Simulators, npj Quantum Inf. 4, 1 (2018).

[50] W. F. Koehl, B. B. Buckley, F. J. Heremans, G. Calusine, and D. D. Awschalom, Room Temperature Coherent Control of Defect Spin Qubits in Silicon Carbide, Nature (London) 479, 84 (2011).

[51] S. Castelletto, B. C. Johnson, V. Ivády, N. Stavrias, T. Umeda, A. Gali, and T. Ohshima, A Silicon Carbide Room-Temperature Single-Photon Source, Nat. Mater. 13, 151 (2014).

[52] S. Buckley, J. Chiles, A. N. McCaughan, G. Moody, K. L. Silverman, M. J. Stevens, R. P. Mirin, S. W. Nam, and J. M. Shainline, All-Silicon Light-Emitting Diodes WaveguideIntegrated with Superconducting Single-Photon Detectors, Appl. Phys. Lett. 111, 141101 (2017).

[53] C. Beaufils, W. Redjem, E. Rousseau, V. Jacques, A. Yu Kuznetsov, C. Raynaud, C. Voisin, A. Benali, T. Herzig, S. Pezzagna, J. Meijer, M. Abbarchi, and G. Cassabois, Optical Properties of an Ensemble of G-Centers in Silicon, Phys. Rev. B 97, 035303 (2018).

[54] A. Brenneis, L. Gaudreau, M. Seifert, H. Karl, M. S. Brandt, H. Huebl, J. A. Garrido, F. H. L. Koppens, and A. W. Holleitner, Ultrafast Electronic Readout of Diamond Nitrogen-Vacancy Centres Coupled to Graphene, Nat. Nanotechnol. 10, 135 (2015).

[55] E. Bourgeois, A. Jarmola, P. Siyushev, M. Gulka, J. Hruby, F. Jelezko, D. Budker, and M. Nesladek, Photoelectric Detection of Electron Spin Resonance of Nitrogen-Vacancy Centres in Diamond, Nat. Commun. 6, 8577 (2015).

[56] M. Morgenstern, V. Gudmundsson, R. Dombrowski, Chr. Wittneven, and R. Wiesendanger, Nonlocality of the
Exchange Interaction Probed by Scanning Tunneling Spectroscopy, Phys. Rev. B 63, 201301 (2001).

[57] N. M. Freitag, L. A. Chizhova, P. Nemes-Incze, C. R. Woods, R. V. Gorbachev, Y. Cao, A. K. Geim, K. S. Novoselov, J. Burgdörfer, F. Libisch, and M. Morgenstern, Electrostatically Confined Monolayer Graphene Quantum Dots with Orbital and Valley Splittings, Nano Lett. 16, 5798 (2016).

[58] A. L. Saraiva, J. Salfi, J. Bocquel, B. Voisin, S. Rogge, R. B. Capaz, M. J. Calderón, and B. Koiller, Donor Wave Functions in Si Gauged by STM Images, Phys. Rev. B 93, 045303 (2016).

[59] K. Sinthiptharakoon, S. R. Schofield, P. Studer, V. Brázdová, C. F. Hirjibehedin, D. R. Bowler, and N. J. Curson, Investigating Individual Arsenic Dopant Atoms in Silicon Using Low-Temperature Scanning Tunnelling Microscopy, J. Phys. Condens. Matter 26, 012001 (2014).

[60] K. Teichmann, M. Wenderoth, S. Loth, R. Ulbrich, J. Garleff, A. Wijnheijmer, and P. Koenraad, Controlled Charge Switching on a Single Donor with a Scanning Tunneling Microscope, Phys. Rev. Lett. 101, 076103 (2008).

[61] J. A. Miwa, J. A. Mol, J. Salfi, S. Rogge, and M. Y. Simmons, Transport through a Single Donor in p-Type Silicon, Appl. Phys. Lett. 103, 043106 (2013).

[62] M. Usman, J. Bocquel, J. Salfi, B. Voisin, A. Tankasala, R. Rahman, M. Y. Simmons, S. Rogge, and L. C. L. Hollenberg, Spatial Metrology of Dopants in Silicon with Exact Lattice Site Precision, Nat. Nanotechnol. 11, 763 (2016).

[63] See Supplemental Material at http://link.aps.org/ supplemental/10.1103/PhysRevX.8.031049 which gives details on the measurements, fitting, model expressions for tunneling spectra and images, and numerical calculations.

[64] M. Rontani and E. Molinari, Imaging Quasiparticle Wave Functions in Quantum Dots via Tunneling Spectroscopy, Phys. Rev. B 71, 233106 (2005).

[65] G. Maruccio, M. Janson, A. Schramm, C. Meyer, T. Matsui, C. Heyn, W. Hansen, R. Wiesendanger, M. Rontani, and E. Molinari, Correlation Effects in Wave Function Mapping of Molecular Beam Epitaxy Grown Quantum Dots, Nano Lett. 7, 2701 (2007).

[66] F. Schulz, M. Ijäs, R. Drost, S. K. Hämäläinen, A. Harju, A. P. Seitsonen, and P. Liljeroth, Many-Body Transitions in a Single Molecule Visualized by Scanning Tunnelling Microscopy, Nat. Phys. 11, 229 (2015).

[67] C. J. Chen, Tunneling Matrix Elements in ThreeDimensional Space: The Derivative Rule and the Sum Rule, Phys. Rev. B 42, 8841 (1990).

[68] T. Ando, Electronic Properties of Two-Dimensional Systems, Rev. Mod. Phys. 54, 437 (1982).

[69] T. B. Boykin, G. Klimeck, M. Friesen, S. N. Coppersmith, P. von Allmen, F. Oyafuso, and S. Lee, Valley Splitting in Low-Density Quantum-Confined Heterostructures Studied Using Tight-Binding Models, Phys. Rev. B 70, 165325 (2004).

[70] S. Goswami, K. A. Slinker, M. Friesen, L. M. McGuire, J. L. Truitt, C. Tahan, L. J. Klein, J. O. Chu, P. M. Mooney, D. W. van der Weide, R. Joynt, S. N. Coppersmith, and 
M. A. Eriksson, Controllable Valley Splitting in Silicon Quantum Devices, Nat. Phys. 3, 41 (2006).

[71] C. H. Yang, A. Rossi, R. Ruskov, N. S. Lai, F. A. Mohiyaddin, S. Lee, C. Tahan, G. Klimeck, A. Morello, and A. S. Dzurak, Spin-Valley Lifetimes in a Silicon Quantum Dot with Tunable Valley Splitting, Nat. Commun. 4, 2069 (2013).

[72] P. Boross, G. Széchenyi, D. Culcer, and A. Pályi, Control of Valley Dynamics in Silicon Quantum Dots in the Presence of an Interface Step, Phys. Rev. B 94, 035438 (2016).

[73] R. Ferdous, E. Kawakami, P. Scarlino, M. P. Nowak, D. R. Ward, D. E. Savage, M. G. Lagally, S. N. Coppersmith, M. Friesen, M. A. Eriksson, L. M. K. Vandersypen, and R. Rahman, Valley Dependent Anisotropic Spin Splitting in Silicon Quantum Dots, npj Quantum Inf. 4, 26 (2018).

[74] W. Huang, M. Veldhorst, N. M. Zimmerman, A. S. Dzurak, and D. Culcer, Electrically Driven Spin Qubit Based on Valley Mixing, Phys. Rev. B 95, 075403 (2017).

[75] J. van Donkelaar, C. Yang, A. D. C. Alves, J. C. McCallum, C. Hougaard, B. C. Johnson, F. E. Hudson, A. S. Dzurak,
A. Morello, D. Spemann, and D. N. Jamieson, Single Atom Devices by Ion Implantation, J. Phys. Condens. Matter 27, 154204 (2015).

[76] J. Luttinger and W. Kohn, Motion of Electrons and Holes in Perturbed Periodic Fields, Phys. Rev. 97, 869 (1955).

[77] A. Tankasala, J. Salfi, J. Bocquel, B. Voisin, M. Usman, G. Klimeck, M. Y. Simmons, L. C. L. Hollenberg, S. Rogge, and R. Rahman, Two-Electron States of a Group-V Donor in Silicon from Atomistic Full Configuration Interactions, Phys. Rev. B 97, 195301 (2018).

[78] C. D. Hill, E. Peretz, S. J. Hile, M. G. House, M. Fuechsle, S. Rogge, M. Y. Simmons, and L. C. L. Hollenberg, A surface Code Quantum Computer in Silicon, Sci. Adv. 1, e1500707 (2015).

[79] J. Towns, T. Cockerill, M. Dahan, I. Foster, K. Gaither, A. Grimshaw, V. Hazlewood, S. Lathrop, D. Lifka, G. D. Peterson, R. Roskies, J. R. Scott, and N. Wilkins-Diehr, XSEDE: Accelerating Scientific Discovery, Comput. Sci. Eng. 16, 62 (2014). 